\title{
Collagen triple helix repeat containing 1 is overexpressed in hepatocellular carcinoma and promotes cell proliferation and motility
}

\author{
MASAHIKO TAMEDA $^{1,2}$, KAZUSHI SUGIMOTO ${ }^{1,2}$, KATSUYA SHIRAKI $^{1}$, NORIHIKO YAMAMOTO ${ }^{1}$, \\ RYUJI OKAMOTO $^{1}$, MASANOBU USUI ${ }^{3}$, MASAAKI ITO ${ }^{1}$, YOSHIYUKI TAKEI ${ }^{4}$, TSUTOMU NOBORI ${ }^{2}$, \\ TAKAHIRO KOJIMA ${ }^{5}$, HIDEAKI SUZUKI ${ }^{6}$, MASAKO UCHIDA ${ }^{6}$ and KAZUHIKO UCHIDA ${ }^{5,6}$ \\ ${ }^{1}$ First Department of Internal Medicine, Departments of ${ }^{2}$ Molecular and Laboratory Medicine, \\ ${ }^{3}$ Hepatobiliary Pancreatic and Transplant Surgery, ${ }^{4}$ Gastroenterology and Hepatology, Mie University School of Medicine, \\ Tsu 514-8507; ${ }^{5}$ Department of Molecular Biological Oncology, Faculty of Medicine, University of Tsukuba, \\ Tsukuba 305-8575; ${ }^{6}$ Research Division, MCBI, Tsukuba 305-0035, Japan
}

Received January 23, 2014; Accepted March 17, 2014

DOI: $10.3892 /$ ijo.2014.2445

\begin{abstract}
Although several therapeutic options are available for hepatocellular carcinoma (HCC), the outcome is still very poor. One reason is the complexity of signal transduction in the pathogenesis of HCC. The aim of this study was to identify new HCC-related genes and to investigate the functions of these genes in the pathogenesis and progression of HCC. Whole genomes of 15 surgically resected HCC specimens were examined for copy number alterations with comparative genomic hybridization. Gene expression was compared between HCC and normal liver tissues. The roles of the new genes in the progression of HCC were studied using cultured cell lines. Copy number gain in chromosome 8q was detected in $53 \%$ of HCC tissues examined. The gene that coded for collagen triple helix repeat containing 1 ( CTHRCI), located at chromosome 8q22.3, was overexpressed in HCC compared with normal or liver cirrhosis tissues and identified as a new HCC-related gene. CTHRC1 deletion with short hairpin RNA significantly reduced proliferation, migration and invasion of HepG2 and Huh7 cells. In addition, mRNA of integrins
\end{abstract}

Correspondence to: Dr Kazushi Sugimoto, First Department of Internal Medicine, Department of Molecular and Laboratory Medicine, Mie University School of Medicine, 2-174 Edobashi, Tsu, Mie 514-8507, Japan

E-mail:kazushi@clin.medic.mie-u.ac.jp

Abbreviations: HCC, hepatocellular carcinoma; CTHRC1, collagen triple helix repeat containing 1 ; $\mathrm{HCV}$, hepatitis $\mathrm{C}$ virus; $\mathrm{HBV}$, hepatitis B virus; CNA, copy number alteration; $\mathrm{CGH}$, comparative genomic hybridization; SNP, single nucleotide polymorphism; qRT-PCR, quantitative real-time polymerase chain reaction; ShRNA, short hairpin RNA

Key words: hepatocellular carcinoma, collagen triple helix repeat containing 1, comparative genomic hybridization, copy number alteration, integrin $\beta$ $\beta-2$ and $\beta-3$ was downregulated, with deletion of CTHRC1 in these cells. Immunohistochemical staining on resected HCC tissues showing positive staining areas for CTHRC1 was significantly greater in poorly-differentiated HCC compared with well-differentiated HCC. Moreover, some cases showed strong staining for CTHRC1 in invasive areas of HCC. CTHRCl has the potential to be a new biomarker for the aggressive HCC, and to be a new therapeutic target in treating HCC.

\section{Introduction}

Hepatocellular carcinoma (HCC) is the most common primary malignancy of the liver in adults. It is the 5 th most frequent cancer worldwide and the 3rd leading cause of cancer mortality (1). Several approaches have been attempted for the treatment of HCC, such as surgical resection, transarterial chemoembolization, radiofrequency ablation and orthotopic liver transplantation. More recently, an oral multikinase inhibitor, sorafenib, has become a key drug for non-resectable HCC (2). Sorafenib inhibits the serine/threonine kinase activity of Raf- 1 and B-Raf, the receptor tyrosine kinase activity of vascular endothelial growth factor receptors (VEGFRs) 1, 2 and 3, and platelet-derived growth factor receptor $\beta$ (PDGFR- $\beta$ ), the cellular signalings of which are implicated in the molecular pathogenesis of HCC.

Despite the variety of therapeutic options, and the many points of action of sorafenib, the prognosis of $\mathrm{HCC}$ is still very poor. Several factors account for the limited efficacy of these treatments. First, most patients have underlying liver disease [e.g., liver cirrhosis due to chronic hepatitis $\mathrm{C}$ virus (HCV) or hepatitis B virus (HBV) infection]. Second, $\mathrm{HCC}$ has a high rate of recurrence that is caused by intrahepatic metastasis or multicentric occurrence. Another reason for its poor outcome is that HCC is a complex and heterogeneous tumor, and many key signal transduction pathways and molecules other than those targeted by sorafenib are possibly implicated in the 
pathogenesis of HCC. In addition, many genetic and epigenetic alterations such as copy number alteration (CNA), DNA methylation, or DNA mutation have been suggested as being related to the development of HCC $(3,4)$. Therefore, it is important to have a clear landscape of the genomic aberrations in order to understand the multistep process of HCC progression.

Among the genetic alterations, CNAs can be found in almost all human malignancies. Several attempts have been made to identify CNAs by searching for new genes that are causative for HCC carcinogenesis. In fact, frequent copy number gains at chromosomes $1 \mathrm{q}, 8 \mathrm{q}$ and $20 \mathrm{q}$, and frequent copy number losses at 1p, 4q, 8p, 16q and 17q have been identified in HCC using array-comparative genomic hybridization (CGH) (5-7). However, the roles of these CNAs in the pathogenesis of HCC have yet to be elucidated.

Given these facts, the purpose of the current study was to identify new HCC-related genes by investigating CNAs in the whole genome using array- $\mathrm{CGH}$, and by focusing on specific genes included in the CNA region. Furthermore, by performing functional assays of the identified genes, we aimed to elucidate the role of the genes in the pathogenesis and progression of $\mathrm{HCC}$, and to clarify whether the genes have the potential to be new therapeutic targets.

\section{Materials and methods}

Patients. Paired tumor and surrounding non-tumor liver tissues (liver cirrhosis) were collected from patients who underwent radical surgery for HCC at Mie University Hospital (Tsu, Japan). Normal liver tissues were obtained from trimmed scrap portions of donor livers used for living donor liver transplantation. In total, liver tissues were obtained from 15 patients and 15 normal subjects. DNA copy number alterations (CNAs) were analyzed in HCC tissues, and gene expression was compared between HCC and normal tissues. Table I shows the patient characteristics. This study was approved by the Institutional Review Board of Mie University Hospital (authorization no. 285). Written informed consent was obtained from each patient included in the study. The study protocol conforms to the ethical guidelines of the 1975 Declaration of Helsinki as reflected in a priori approval by the institution's human research committee.

Copy number analysis. GeneChip 50K single nucleotide polymorphism (SNP) mapping array analysis was performed according to the standard Single Primer GeneChip Mapping Assay protocol using a Human Mapping 50K Array Hind III (Affymetrix, Santa Clara, CA, USA). Individual SNP copy numbers and chromosomal regions with gains or deletions were evaluated with CNAG 2.0 (8).

Expression profiling. Oligonucleotide microarray experiments were carried out using Human Genome U133 Plus 2.0 arrays according to the manufacturer's instructions (Affymetrix). Data were analyzed with GeneSpring GX 7.3.1 (Silicon Genetics, Redwood City, CA, USA).

HCC cell lines. The human HCC cell lines HepG2 (RCB1648) and Huh7 (RCB1942) were purchased from the Riken Cell Bank (Tsukuba, Japan), Hep3B (ATCC HTB-52) and SK-Hep1
Table I. Characteristics of HCC patients studied.

\begin{tabular}{llc}
\hline Gender & Male & 11 \\
& Female & 4 \\
Age & & $62 \pm 8.7^{\mathrm{a}}$ \\
HBV serology & Positive & 7 \\
& Negative & 8 \\
HCV serology & Positive & 8 \\
& Negative & 7 \\
AFP $(\mathrm{ng} / \mathrm{ml})$ & & $885 \pm 3,107^{\mathrm{a}}$ \\
DCP $(\mathrm{mAU} / \mathrm{ml})$ & & $2,168 \pm 6,243^{\mathrm{a}}$ \\
\end{tabular}

${ }^{\mathrm{a}}$ Mean $\pm \mathrm{SD}$. HBV, hepatitis B virus; HCV, hepatitis C virus; AFP, $\alpha$-fetoprotein; DCP, des- $\gamma$-carboxy prothrombin.

(ATCC HB-8064) were purchased from the American Type Culture Collection (Manassas, VA), and HLE (JCRB0404) and PLC/PRF/5 (JCRB0406) were purchased from the Health Science Research Resources Bank (Osaka, Japan). All cell lines were cultured in Dulbecco's modified Eagle's medium (DMEM) (Life Technologies, Tokyo, Japan) supplemented with $1 \%$ penicillin/streptomycin (Life Technologies) and $10 \%$ fetal calf serum (FCS) (Life Technologies) in a humidified atmosphere containing $5 \% \mathrm{CO}_{2}$ at $37^{\circ} \mathrm{C}$.

Qualitative reverse transcription polymerase chain reaction $(P C R)$. The expression of CTHRC1 mRNA in the HCC cell lines was determined by reverse transcription PCR of total RNA. Total RNA was extracted from approximately $10^{7}$ cells of each cell line with the RNeasy mini kit (Qiagen, Tokyo, Japan), and cDNA was synthesized by extension of oligo dT primers with PrimeScript reverse transcriptase (Takara Bio, Inc., Otsu, Japan). PCR of the cDNA was performed with Ex Taq (Takara Bio). The sense primer used for amplification of CTHRC1 was 5'-AGGGAGGTGGTGGACCTGTAT-3' and antisense primer was 5'-GCCAACCCAGATAGCAACAT-3'.

Quantitative real-time PCR. The cDNA of HCC tissues, non-tumorous tissues and HCC cell lines was synthesized from $1 \mu \mathrm{g}$ of total RNA and quantitative real-time PCR (qRT-PCR) was performed using the ABI prism 7300 Real-time PCR system (Applied Biosystems, Foster City, CA, USA) with EagleTaq master mix kits (Roche Molecular Systems, Branchburg, NJ, USA). The expression levels of target genes from triplicate reactions were determined by normalization to $\beta$-actin according to the manufacturer's instructions. Primer sets are as follows: CTHRC1 forward, 5'-CCAAGGGGAAGCAAAAGG-3'; reverse, 5'-CCCTTGTAAGCACATTCCATTA-3'. Human integrin $\beta$-2 forward, 5'-CAGCAATGTGGTCCAACTCA-3'; reverse, 5'-GAGGGCGTTGTGATCCAG-3'. Human integrin $\beta$-3 forward, 5'-CGCTAAATTTGAGGAAGAACG-3'; reverse, 5'-GAAGGTAGACGTGGCCTCTTT-3'.

Western blot analysis. Polyclonal antibody for CTHRC1 was generated by immunization of rabbits. HepG 2 cells were fractionated using the ProteoExtract Subcellular Proteome Extraction Kit (Merck Millipore, Darmstadt, Germany) according to the manufacturer's instructions, and localiza- 
tion of CTHRC1 in HCC cells was determined by western blot analysis. Protein lysates of each fraction were separated by SDS-polyacrylamide gel electrophoresis (12.5\%) and transferred to polyvinylidene difluoride membranes. Blots were blocked with $5 \%$ milk in Tris- $\mathrm{HCl}(\mathrm{pH} 7.5)$ with $0.1 \%$ Tween-20 for $2 \mathrm{~h}$ and proved with primary antibody at $4^{\circ} \mathrm{C}$ overnight. The immunoblots were then probed with horseradish peroxidase-conjugated anti-rabbit secondary antibody (GE Healthcare, Amersham Place, UK) and visualized using ECL plus (GE Healthcare, Munich, Germany).

Knockdown of CTHRC1 mRNA. Three types of short hairpin RNA (ShRNA) against CTHRC1 and control ShRNA were constructed using the piGENE vector (Igene, Tokyo, Japan). Their target sequences are listed as follows: Sh1, GAAATGA ATTCAACAATTA; Sh2, AAGGAAGCCCTGAAATGAA; Sh3, AGGGAAAGCTTTGAGGAGT; and control (T7STOP), CACCTTTTTTTT. These ShRNAs and control plasmid were transfected into HepG2 cells and Huh7 cells with FuGENE HD (Roche, Mannheim, Germany), followed by the addition of $1 \mu \mathrm{g} / \mathrm{ml}$ of puromycin after $24 \mathrm{~h}$ for selecting transfected cells. Cells were harvested $72 \mathrm{~h}$ later for analysis of gene expression, cell proliferation, migration and invasion.

Cell proliferation assay. Cell proliferation was assessed with the xCELLigence system (Roche Inc., Basel, Switzerland) according to the manufacturer's instructions. Briefly, each well of a 16-well microtiter plate (E-Plate 16) was filled with $100 \mu 1$ of DMEM to equilibrate the well membrane, and each plate was incubated for $30 \mathrm{~min}$ at $37^{\circ} \mathrm{C}$ in $5 \% \mathrm{CO}_{2}$. $\mathrm{HCC}$ cells transfected with ShRNA against CTHRC1 or control plasmid were suspended in $100 \mu 1$ of DMEM and seeded at a density of $1 \times 10^{4}$ cells per well. Cells were cultured for $48 \mathrm{~h}$ with the Real-Time Cell Analyzer (RTCA) single plate (SP) instrument placed in a standard incubator at $37^{\circ} \mathrm{C}$ in $5 \% \mathrm{CO}_{2}$. Then, cell proliferation was monitored by recording cell index $(\mathrm{CI})$ values at 15 -min intervals for $48 \mathrm{~h}$.

Cell migration and invasion assays. Cell migratory and invasive abilities were also assessed by the xCELLigence system. Briefly, cell migration was assessed by seeding HCC cells at $2 \times 10^{4} /$ well on a fibronectin-coated CIM-16 plate, and cell invasion was assessed by seeding HCC cells at $3 \times 10^{4} /$ well on a CIM-16 plate coated with Matrigel (Becton-Dickinson, Tokyo, Japan). Both cell invasion and migration were monitored by recording the CI at 15-min intervals for $48 \mathrm{~h}$.

Real-time PCR array. The changes in gene expression related to cell migration and invasion by CTHRC1 knockdown in HCC cells were analyzed with the Human Extracellular Matrix and Adhesion Molecules RT2 Profiler PCR Array (SABiosciences, Frederick, MD, USA) according to the manufacturer's instructions. These changes were confirmed also by qRT-PCR.

Immunohistochemistry. Immunohistochemical staining for CTHRC1 was performed on surgically resected HCC tissues using the Vectastain ABC kit (Vector Laboratories, Burlingame, CA, USA). Deparaffinized sections were heated for $5 \mathrm{~min}$ in citrate buffer at $100^{\circ} \mathrm{C}$ with a pressure cooker to
Table II. Chromosomes which had frequent copy number alterations in $\mathrm{HCC}$.
Gain
Loss

Chromosome Frequency (\%) Chromosome Frequency (\%)

\begin{tabular}{rrrr}
\hline $1 \mathrm{q}$ & 60 & $17 \mathrm{p}$ & 47 \\
$8 \mathrm{q}$ & 53 & $8 \mathrm{p}$ & 27 \\
$13 \mathrm{q}$ & 27 & $16 \mathrm{q}$ & 27 \\
$20 \mathrm{q}$ & 27 & $1 \mathrm{p}$ & 20 \\
$6 \mathrm{p}$ & 27 & $2 \mathrm{q}$ & 20 \\
$17 \mathrm{q}$ & 20 & $13 \mathrm{q}$ & 20 \\
\hline
\end{tabular}

reactivate the antigen, and treated with $0.3 \% \mathrm{H}_{2} \mathrm{O}_{2}$ in methanol for $30 \mathrm{~min}$ to abolish endogenous peroxidase activity. Sections were blocked with $1 \%$ goat serum in phosphate-buffered saline, covered with primary antibody at $4^{\circ} \mathrm{C}$ overnight, and then covered with second-step biotinylated antibody for $30 \mathrm{~min}$, and incubated with peroxidase-labeled streptavidin for $30 \mathrm{~min}$. After washing, sections were incubated with $0.05 \%$ diaminobenzidine $/ 0.15 \% \quad \mathrm{H}_{2} \mathrm{O}_{2}$ and counterstained with $10 \%$ hematoxylin (Wako Pure Chemical Industries, Ltd., Osaka, Japan).

Statistical analysis. Comparisons of gene expression, cell proliferation, migration and invasion were performed using the two-tailed Student's t-test. For differences between rates, Fisher's exact test was used. $\mathrm{p}<0.05$ was considered statistically significant.

\section{Results}

CTHRC1 was identified as a new HCC-related gene. The chromosomes which had frequent CNAs ( $\geq 20 \%)$ in $15 \mathrm{HCC}$ tissues are listed in Table II. Among them, we focused on 8q, in which CNAs were detected in $53 \%$ of HCC tissues, because CNAs in $8 q$ have been also reported very frequently by several groups (9-11), but have not been studied in detail. Through gene expression profiling of $8 \mathrm{q}$, we identified CTHRC1 located at $8 \mathrm{q} 22.3$ as a new HCC-related gene, of which the expression level was higher in HCC compared with normal tissues by 13.5-fold (Fig. 1A). Moreover, re-validation with qRT-PCR revealed that expression levels of CTHRC1 mRNA increased from normal tissues to liver cirrhosis and $\mathrm{HCC}$ in a stepwise manner (Fig. 1B). We investigated the expression of CTHRC1 mRNA in HCC cell lines by RT-PCR. As shown in Fig. 1C, CTHRC1 transcripts were detected in all 6 HCC cell lines. Localization of the CTHRC1 protein in HCC cells was also investigated using HepG2. HepG2 cells were fractionated and each fraction was analyzed for CTHRC1 expression by western blot analysis. Fig. 1D shows that the $26-\mathrm{kDa}$ CTHRC1 protein was expressed in membrane fractions of the HepG2 cells.

CTHRC1 promotes proliferation of HCC cells. As it has been reported that CTHRC1 is involved in tissue remodeling in 
A

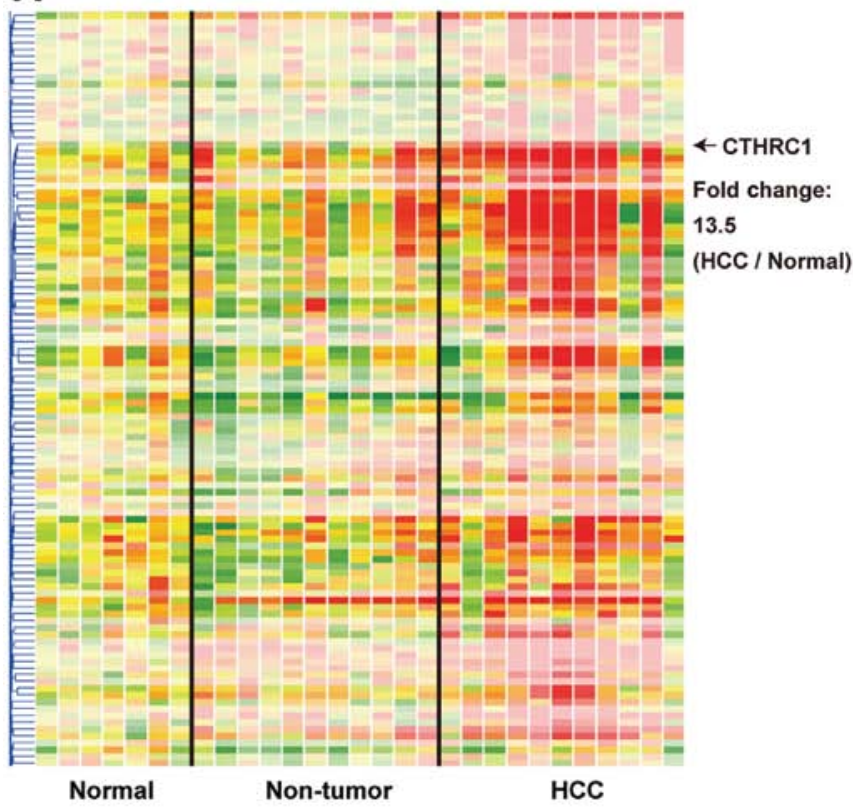

B
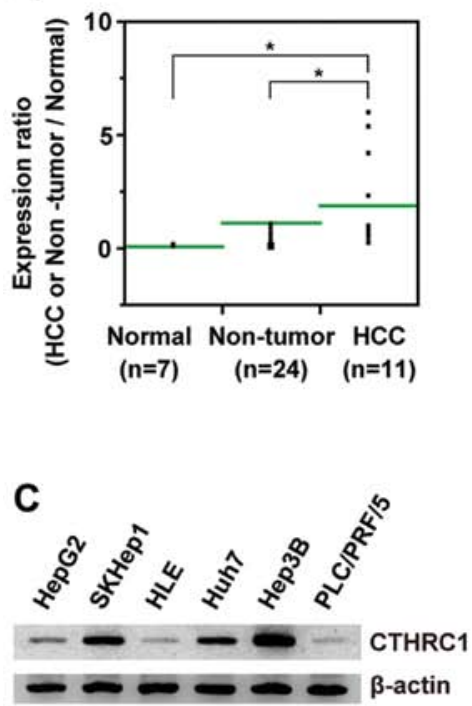

Figure 1. Results of expression analyses. (A) Gene expression profiling of chromosome 8q. CTHRC1 located at 8q22.3 of which expression level was 13.5-fold higher in HCC compared with normal liver tissues was identified as a new HCC-related gene. (B) Expression levels of CTHRC1 mRNA in each tissue. The expression levels of CTHRC1 mRNA increased from normal tissues to liver cirrhosis and HCC in a stepwise manner $(" \mathrm{p}<0.05)$. (C) Expression of CTHRC1 mRNA in HCC cell lines. (D) Expression of CTHRC1 in each cell fraction assessed by western blot analysis. A 26-kDa CTHRC1 protein was detected in membrane fractions.

rheumatoid arthritis and injured arteries by promoting the migration of fibroblasts (12), we hypothesized that CTHRC1 might have some role in the proliferation and motility of HCC cells. First, we investigated the effect of CTHRC1 on the proliferation of HCC cells. Three types of ShRNA were used to suppress the expression of CTHRC1 in HepG2 and Huh7 cells. The knockdown efficiency was confirmed by qRT-PCR (Fig. 2A and B). As shown in Fig. 2C and D, cell proliferation after 24 and $48 \mathrm{~h}$ assessed with xCELLignece system was
A

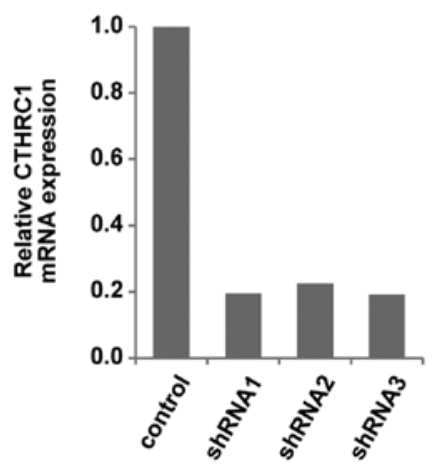

B

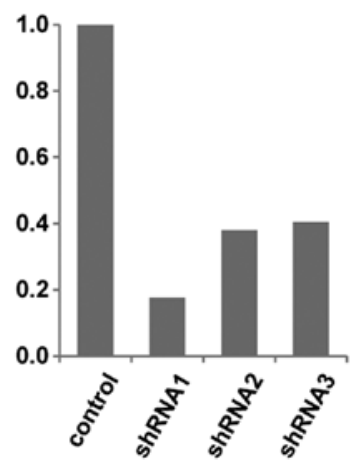

C
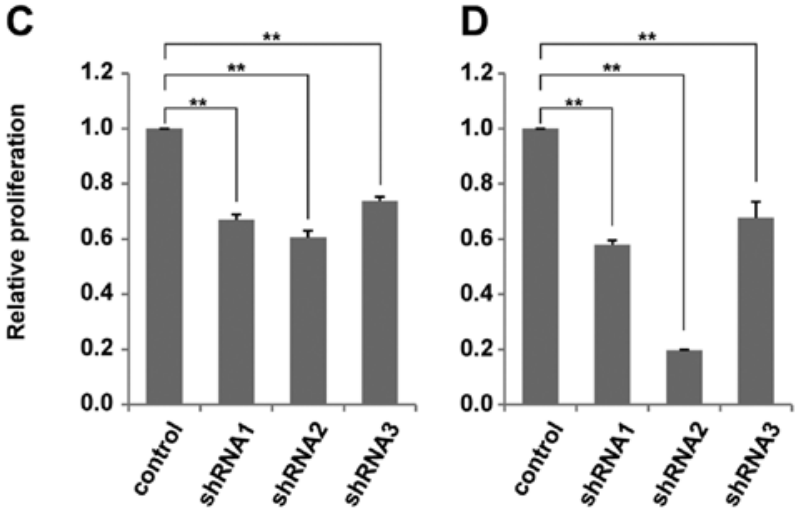

E

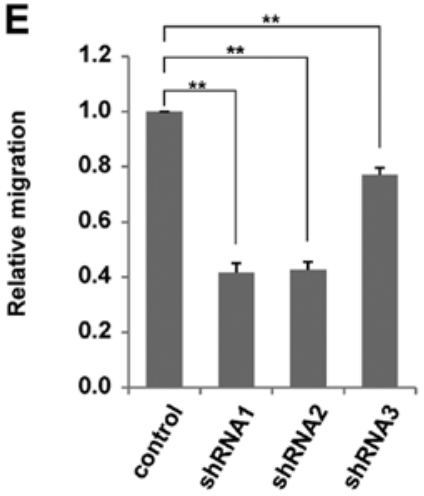

$\mathbf{F}$

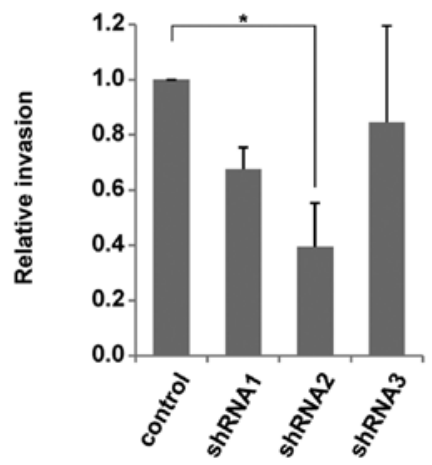

G

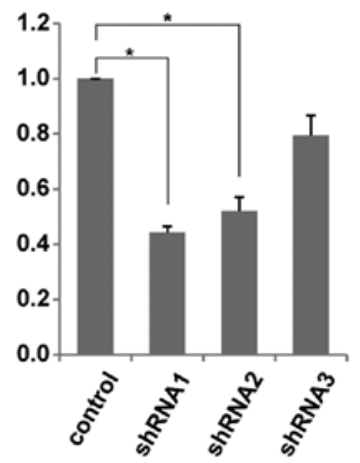

Figure 2. Functional analyses following CTHRC1-deletion in HCC cells Inhibition of CTHRC1 mRNA in (A) HepG2 and (B) Huh7 with short hairpin (ShRNA). Cell proliferation of (C) HepG2 and (D) Huh7. Cell proliferation after 24 and $48 \mathrm{~h}$ was significantly decreased in both the HepG2 and Huh7 cells with CTHRC1 knockdown compared with the control cells. (E) Cell migration of Huh7. Cell invasion of (F) HepG2 and (G) Huh7. Both cell migration and invasion were significantly reduced by CTHRC1 knockdown. The data are expressed as the mean $\pm \mathrm{SD}$ relative to control of five independent experiments $\left(\mathrm{p}<0.05,{ }^{* * *} \mathrm{p}<0.01\right)$. 
A
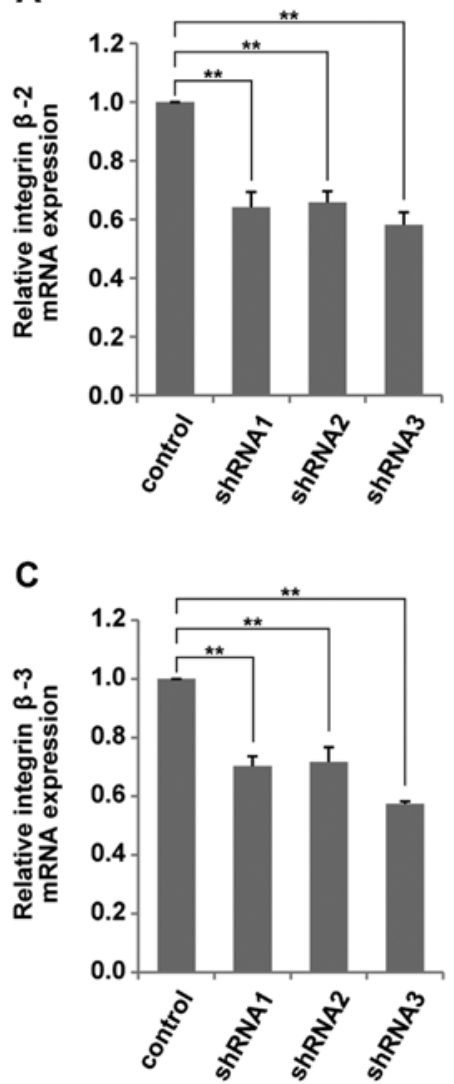

B

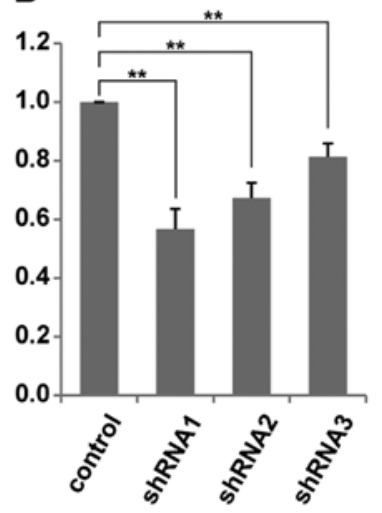

D

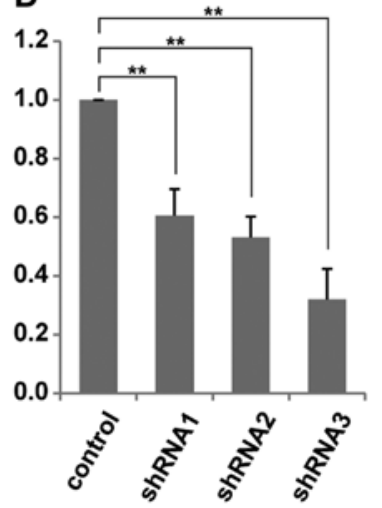

Figure 3. Expression of integrin mRNA following CTHRC1-deletion in HCC cells. (A) Expression of integrin $\beta-2$ in HepG2 and (B) Huh7. Expression level of integrin $\beta-2$ mRNA was significantly decreased with CTHRC1-depletion in HCC cells. (C) Expression of integrin $\beta-3$ in HepG2 and (D) Huh7. Expression level of integrin $\beta-3$ mRNA was also significantly decreased with CTHRC1-depletion in HCC cells. The data are expressed as the mean \pm SD relative to control of five independent experiments $\left({ }^{* *} \mathrm{p}<0.01\right)$.

significantly decreased in both the HepG2 and Huh7 cells with CTHRC1 knockdown compared with the control cells.

CTHRCl promotes migration and invasion of HCC cells. We next examined the influence of CTHRC1 knockdown on HCC cell migratory activity. As shown in Fig. 2E, CTHRC1 knockdown caused a significant reduction in cell migration after $24 \mathrm{~h}$ in the Huh7 cells compared with the control cells. In addition, the results of the cell invasion assay using the Matrigel-coated plate showed that the cell invasions of both the HepG2 and Huh7 cells after $24 \mathrm{~h}$ were also significantly reduced in the CTHRC1-depleted cells compared with the control cells (Fig. 2F and G).

CTHRCl knockdown reduces integrin $\beta$ mRNA in HCC cells. To elucidate the mechanism of the suppression of cell migratory and invasive activity in HCC cells by CTHRC1 knockdown, we compared the mRNA expression, which related to cell migration and invasion between the CTHRC1-depleted HepG2 cells and the control cells, using real-time PCR array. The results suggested that the expression of integrin $\beta$ was affected by suppression of CTHRC1 mRNA (data not shown). To confirm these results, we next performed qRT-PCR to examine the changes in the expression levels of integrin $\beta$ mRNA in HCC

Table III. Summary of immunohistochemical staining.

\begin{tabular}{lcccc}
\hline & & \multicolumn{3}{c}{ Extent of staining area } \\
\cline { 3 - 5 } & Case & $0-33 \%$ & $34-66 \%$ & $67-100 \%$ \\
\hline Total HCC & 41 & 36.6 & 24.4 & 39.0 \\
Well diff & 11 & 63.6 & 18.2 & 18.2 \\
Mod diff & 23 & 26.1 & 34.8 & 39.1 \\
Poorly diff & 7 & 28.6 & 0 & $71.4^{\text {a }}$ \\
\hline
\end{tabular}

Well diff, well-differentiated HCC; mod diff, moderately-differentiated HCC; poorly diff, poorly-differentiated HCC. ${ }^{a} \mathrm{p}<0.05$ vs. well diff.

cells after knockdown of CTHRC1. As shown in Fig. 3, mRNA expressions of integrin $\beta-2$ and integrin $\beta-3$ were significantly reduced with CTHRC1 depletion in both the HepG2 and Huh7 cells. On the other hand, no cell proliferation, migration/invasion, or amount of integrin $\beta$ mRNA in HCC changed with overexpression of CTHRC1 (data not shown).

CTHRCl is expressed in the invasive area of HCC tissues. Forty-one surgically resected HCC specimens and adjacent non-tumor tissues were examined for CTHRC1 expression by immunohistochemistry. The HCC tissues were divided into three groups according to their degree of differentiation: well-differentiated HCC, moderately-differentiated HCC and poorly-differentiated HCC. The levels of CTHRC1 expression were analyzed in each group and the percentages of positive staining area were divided into three categories. The results are summarized in Table III. The rate for the positive area for CTHRC1 greater than $66 \%$ was significantly higher in poorly-differentiated HCC than that of well-differentiated HCC ( 71.4 vs. $18.2 \%, p<0.05)$. The differences of these rates in both between well-differentiated HCC and moderatelydifferentiated HCC, and between moderately-differentiated HCC and poorly-differentiated HCC were not significant, probably due to the small sample number. None of the patients had positive CTHRC1 staining in the adjacent non-tumorous areas. Interestingly, some patients showed strong positive staining for CTHRC1 in the HCC cells close to fibrous boundaries (Fig. 4) and in invasive areas (Fig. 4B, arrowheads), suggesting high migratory and invasive activity of these cells.

\section{Discussion}

Despite the availability of several therapeutic options, it is still difficult to control the progression of HCC completely. One reason is the complexity of signal transduction in HCC; in other words, a large number of molecules are involved in the pathogenesis of HCC. Therefore, it is important to search for the novel molecules related to HCC progression in order to understand the mechanism of its pathogenesis and to achieve better prognosis. In this study, we identified CTHRCl located at chromosome $8 \mathrm{q} 22.3$ as a novel HCC-related gene. The results of functional assay showed that CTHRC1-deletion caused reduced cell proliferation and motility in HCC cells. 

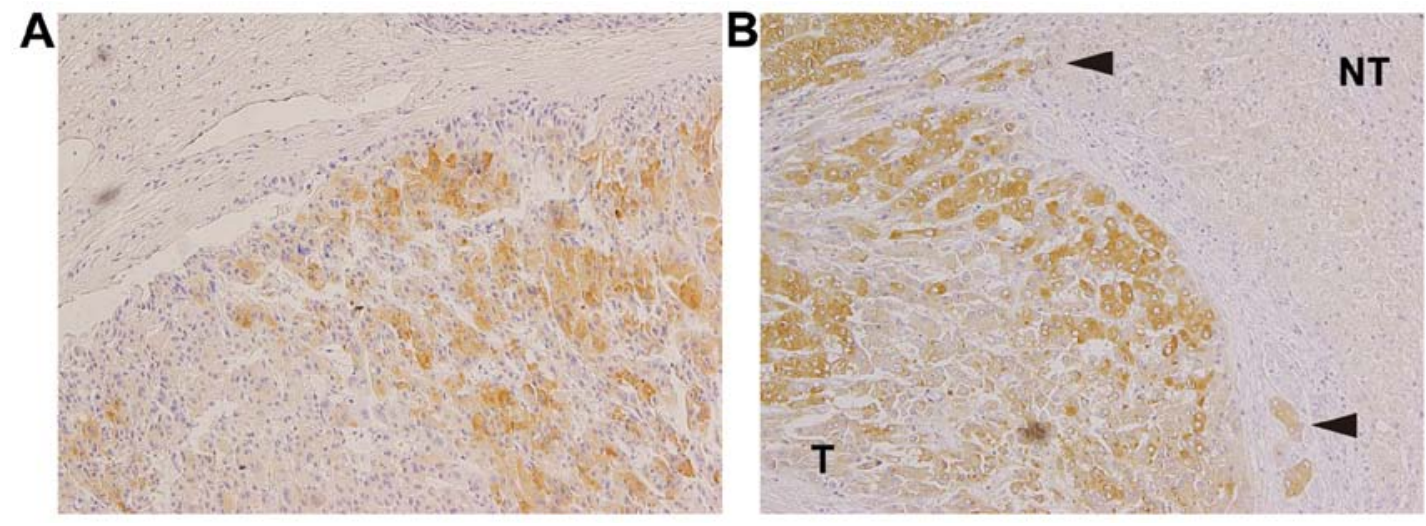

Figure 4. Immunohistochemistry for CTHRC1 on HCC tissues. (A and B) Strong positive staining for CTHRC1 was observed in the HCC cells close to fibrous boundaries, while non-tumor parts were negative for CTHRC1. (B, arrowheads) HCC cells invading surrounding normal liver tissues were strongly positive for CTHRC1. T, tumor; NT, non-tumor. Original magnification, x200.

In addition, integrin $\beta$ mRNA expression was decreased by knockdown of CTHRC1 in HCC cells. Moreover, the CTHRC1 protein was overexpressed in HCC tissues, especially in poorly-differentiated HCCs.

We first analyzed the whole genome for CNA using array-CGH and found copy number gain of $8 \mathrm{q}$ in $53 \%$ of the HCC tissues. We next performed gene expression analysis of $8 \mathrm{q}$ and identified $C T H R C 1$ as a novel HCC-related gene. Our results showed that CTHRC1 was overexpressed in HCC tissues compared with the surrounding non-tumor tissues. Furthermore, CTHRC1 mRNA was upregulated in all HCC cell lines examined, and the CTHRC1 protein was located in the cell membranes of the HCC cells. The mammalian CTHRCl gene was first identified in balloon-injured arteries by Pyagay et al (12). This gene is highly conserved among vertebrates. The CTHRC1 protein contains an $\mathrm{NH} 2$-terminal peptide for extracellular secretion, a short collagen triple helix repeat of 36 amino acids, and a $\mathrm{COOH}$-terminal globular domain. Although CTHRC1 expression was not detectable in normal arteries, on injury, it was transiently expressed by fibroblasts of the remodeling adventitia and by smooth muscle cells of the neointima. Durmus et al (13) reported that CTHRC1 was highly expressed in developing bones and cartilage, the bone matrix and periosteum of adult bones, and in the epithelium-mesenchymal interface including epidermis, airway epithelium, and esophageal epithelium. In addition, these sites of CTHRC1 expression were found to overlap considerably with those reported for the transforming growth factor $\beta$ (TGF- $\beta$ ) family members and interstitial collagens (13). Kimura et al also reported that CTHRC1 increased bone mass as a positive regulator of postnatal bone formation (14). These investigators found that CTHRC1-null mice had a decreased number of osteoblasts and low bone mass, while CTHRC1 transgenic mice had an increased number of osteoblasts and displayed high bone mass. Furthermore, expression of CTHRC1 has been reported in stromal cells of breast cancer (15). Using cDNA arrays, Tang et al suggested that the CTHRC1 gene was aberrantly expressed in several types of human solid cancer cells, especially in cancers of the gastrointestinal tract, lung, breast, thyroid, ovarian, cervix, liver and pancreas (16).
The results of the present study were congruent with the report by Tang et al (16). Although it is still unclear whether CTHRC1 has any function in carcinogenesis, several reports have suggested that inflammation and tissue repair are tightly linked with the development of cancer $(17,18)$. Therefore, it is possible that CTHRC1 plays a certain role in the early stage of carcinogenesis of several cancers, including HCC.

To further elucidate the potential roles of CTHRC1 in HCC progression, we next analyzed the effect of CTHRC1 deletion on the proliferation, migration and invasion of HCC cells. Our results showed that HCC cell proliferation was reduced by the deletion of CTHRC1. Moreover, knockdown of CTHRC1 also reduced the migratory and invasive ability of HCC cells. Several previous studies have suggested that CTHRC1 is related to the motility and invasion of both non-tumor and tumor cells. Using a microarray, Turashvili et al found that the CTHRC1 gene was upregulated in invasive lobular breast carcinoma, suggesting the relation of CTHRC1 to carcinogenesis and cancer progression (19). Using the scratch wound healing assay, Pyagay et al showed that both PAC1 cells and embryonic fibroblasts overexpressing CTHRC1 increased migratory activities compared with control cells (12). Tang et al reported that CTHRC1 expression was significantly higher in invasive melanoma than in non-invasive melanoma (16). In addition, the experimental results of Tang et al with the Boyden chamber also showed that when CTHRC1 expression was inhibited with small interfering RNA (siRNA), migration of melanoma cells was significantly reduced (16). Our experimental results with the real-time cell analyzer indicated that CTHRC1 plays important roles, not only in proliferation or migration, but also in invasion of HCC cells, supporting the findings of previous reports. Therefore, there is a possibility that $C T H R C 1$ is a new target for therapy of $\mathrm{HCC}$ especially for preventing metastasis.

Next, to elucidate the mechanism of how CTHRCl affects the motility of HCC cells, we analyzed the changes in mRNA expression. The results of the mRNA array suggested that expression of integrin $\beta-2$ and $\beta-3$ mRNA was reduced by CTHRC1 deletion; this was confirmed by qRT-PCR. Integrins are a superfamily of transmembrane receptors that form heterodimers of $\alpha$ - and $\beta$-subunits. By binding to extracellular matrix (ECM) components, integrins mediate 
cell adhesion and direct a number of cellular processes such as proliferation, migration and differentiation (20-22). Integrins are also important in promoting cell survival by preventing anoikis, which is apoptosis induced by anchorage-dependent cells detaching from the surrounding ECM $(23,24)$. There has been accumulating evidence showing that integrins $\beta-2$ and $\beta-3$ have a relationship with cancer progression and migration, including HCC (25-31). For example, Li et al reported that the downregulation of integrin $\beta-3$ in small cell lung cancer cells reduced cell migration/invasion and induced apoptosis (30). Our results suggest that CTHRC1 promotes the migration/invasion of HCC cells, and also increases cell survival of HCC by inhibiting anoikis via integrin $\beta$ expression. However, no cell proliferation, migration/invasion, or amount of integrin $\beta$ mRNA in HCC changed with overexpression of CTHRC1 (data not shown). Presumably, the expression level of CTHRC1 in HCC cells is already sufficient; therefore, increasing its expression does not affect these cell processes to any greater degree. In addition, the detailed mechanism on how CTHRC1 regulates the expression of integrin $\beta$ remains to be elucidated.

Another possible mechanism by which CTHRC1 mediates cell motility is activation of Wnt signaling. Yamamoto et al found that CTHRC1 is a Wnt cofactor protein that selectively activates the planar cell polarity (PCP) pathway by stabilizing ligand-receptor interaction (32). The PCP pathway of Wnt signaling is mediated by the activation of small GTP-binding proteins, including Rac, Rho, Jun N-terminal kinase and Rho-associated kinase, and regulates actin polymerization and cell migration $(33,34)$. Therefore, it is possible that CTHRC1 promotes cell migration through activation of the PCP pathway of Wnt signaling.

Finally, our results of immunohistochemical staining for CTHRC1 showed that poorly-differentiated HCC had a higher expression level of CTHRC1 compared with welldifferentiated HCC, supporting previous findings in invasive breast cancer (19). Moreover, strongly positive staining was observed in tumor cells around the cancer borderlines or invasive areas in several cases, suggesting that those cells had a higher activity level of proliferation and migration. These results indicate that $C T H R C 1$ can be a new biomarker for aggressive HCC.

During preparation of this manuscript, Park et al reported the roles of CTHRC1 in pancreas cancer (35) and very recently, Chen et al also reported CTHRC1 expression in HCC (36). They found CTHRC1 increased motility and adhesiveness of pancreas cancer cells and HCC cells. Moreover, Chen et al also reported HCC with higher CTHRC1 mRNA expression had worse prognosis (36). Our results are clearly supporting these findings. In addition, our data add the information that CTHRC1 protein is actually expressed in human HCC tissues, more prominently in cancer cells which are supposed to be aggressive.

Taken together, the results of the present study obtained from cultured cell lines and human HCC tissues indicated that CTHRC1 is upregulated in HCC cells and promotes cell proliferation, migration and invasion. CTHRC1 has the potential to be a new biomarker for types of $\mathrm{HCC}$ with poor prognosis, and to be a new therapeutic target for HCC.

\section{Acknowledgements}

We thank Mina Tenpaku and Hiromi Nishii for their technical assistance.

\section{References}

1. Bosch FX, Ribes J, Diaz M and Cleries R: Primary liver cancer: worldwide incidence and trends. Gastroenterology 127: S5-S16, 2004.

2. Llovet JM, Ricci S, Mazzaferro V, et al: Sorafenib in advanced hepatocellular carcinoma. N Engl J Med 359: 378-390, 2008.

3. Aleksic K, Lackner C, Geigl JB, et al: Evolution of genomic instability in diethylnitrosamine-induced hepatocarcinogenesis in mice. Hepatology 53: 895-904, 2011.

4. Neumann O, Kesselmeier M, Geffers R, et al: Methylome analysis and integrative profiling of human HCCs identify novel protumorigenic factors. Hepatology 56: 1817-1827, 2012.

5. Nishida N, Nishimura T, Ito T, Komeda T, Fukuda $Y$ and Nakao K: Chromosomal instability and human hepatocarcinogenesis. Histol Histopathol 18: 897-909, 2003.

6. Patil MA, Gutgemann I, Zhang J, et al: Array-based comparative genomic hybridization reveals recurrent chromosomal aberrations and Jab1 as a potential target for 8q gain in hepatocellular carcinoma. Carcinogenesis 26: 2050-2057, 2005.

7. Katoh H, Ojima H, Kokubu A, et al: Genetically distinct and clinically relevant classification of hepatocellular carcinoma: putative therapeutic targets. Gastroenterology 133: 1475-1486, 2007.

8. Nannya Y, Sanada M, Nakazaki K, et al: A robust algorithm for copy number detection using high-density oligonucleotide single nucleotide polymorphism genotyping arrays. Cancer Res 65: 6071-6079, 2005.

9. Collonge-Rame MA, Bresson-Hadni S, Koch S, et al: Pattern of chromosomal imbalances in non-B virus related hepatocellular carcinoma detected by comparative genomic hybridization. Cancer Genet Cytogenet 127: 49-52, 2001.

10. Chochi Y, Kawauchi S, Nakao M, et al: A copy number gain of the $6 \mathrm{p}$ arm is linked with advanced hepatocellular carcinoma: an array-based comparative genomic hybridization study. J Pathol 217: 677-684, 2009.

11. Midorikawa Y, Yamamoto S, Ishikawa S, et al: Molecular karyotyping of human hepatocellular carcinoma using singlenucleotide polymorphism arrays. Oncogene 25: 5581-5590, 2006.

12. Pyagay $\mathrm{P}$, Heroult $\mathrm{M}$, Wang $\mathrm{Q}$, et al: Collagen triple helix repeat containing 1 , a novel secreted protein in injured and diseased arteries, inhibits collagen expression and promotes cell migration. Circ Res 96: 261-268, 2005.

13. Durmus T, LeClair RJ, Park KS, Terzic A, Yoon JK and Lindner V: Expression analysis of the novel gene collagen triple helix repeat containing-1 (Cthrc1). Gene Expr Patterns 6: 935-940, 2006

14. Kimura $\mathrm{H}$, Kwan $\mathrm{KM}, \mathrm{Zhang} \mathrm{Z}$, et al: $\mathrm{Cthrc1}$ is a positive regulator of osteoblastic bone formation. PLoS One 3: e3174, 2008.

15. West RB, Nuyten DS, Subramanian S, et al: Determination of stromal signatures in breast carcinoma. PLoS Biol 3: e187, 2005.

16. Tang L, Dai DL, Su M, Martinka M, Li G and Zhou Y: Aberrant expression of collagen triple helix repeat containing 1 in human solid cancers. Clin Cancer Res 12: 3716-3722, 2006.

17. Coussens LM and Werb Z: Inflammation and cancer. Nature 420: 860-867, 2002.

18. Beachy PA, Karhadkar SS and Berman DM: Tissue repair and stem cell renewal in carcinogenesis. Nature 432: 324-331, 2004.

19. Turashvili G, Bouchal J, Baumforth K, et al: Novel markers for differentiation of lobular and ductal invasive breast carcinomas by laser microdissection and microarray analysis. BMC Cancer 7: $55,2007$.

20. Oktay M, Wary KK, Dans M, Birge RB and Giancotti FG: Integrin-mediated activation of focal adhesion kinase is required for signaling to Jun $\mathrm{NH} 2$-terminal kinase and progression through the G1 phase of the cell cycle. J Cell Biol 145: 1461-1469, 1999.

21. Clark EA and Brugge JS: Integrins and signal transduction pathways: the road taken. Science 268: 233-239, 1995.

22. Giancotti FG and Ruoslahti E: Integrin signaling. Science 285: 1028-1032, 1999. 
23. Maubant S, Saint-Dizier D, Boutillon M, et al: Blockade of alpha $\mathrm{v}$ beta 3 and alpha $\mathrm{v}$ beta5 integrins by RGD mimetics induces anoikis and not integrin-mediated death in human endothelial cells. Blood 108: 3035-3044, 2006.

24. Benoit YD, Larrivee JF, Groulx JF, Stankova J, Vachon PH and Beaulieu JF: Integrin alpha8beta1 confers anoikis susceptibility to human intestinal epithelial crypt cells. Biochem Biophys Res Commun 399: 434-439, 2010.

25. Takeichi T, Mocevicius P, Deduchovas O, et al: Alpha v beta2 integrin is indispensable for $\mathrm{CD}^{+} \mathrm{T}$-cell recruitment in experimental pancreatic and hepatocellular cancer. Int J Cancer 130: 2067-2076, 2012.

26. Oberyszyn TM, Conti CJ, Ross MS, et al: Beta2 integrin/ICAM-1 adhesion molecule interactions in cutaneous inflammation and tumor promotion. Carcinogenesis 19: 445-455, 1998.

27. Wu Y, Zuo J, Ji G, et al: Proapoptotic function of integrin beta(3) in human hepatocellular carcinoma cells. Clin Cancer Res 15: 60-69, 2009.

28. Jeanes AI, Wang P, Moreno-Layseca P, et al: Specific beta-containing integrins exert differential control on proliferation and two-dimensional collective cell migration in mammary epithelial cells. J Biol Chem 287: 24103-24112, 2012.

29. Jung CW, Song TJ, Lee KO, et al: Characterization of hepatocellular carcinoma cell lines based on cell adhesion molecules. Int J Mol Med 29: 1158-1164, 2012.
30. Li N, Zhang JP, Guo S, et al: Down-regulation of beta3-integrin inhibits bone metastasis of small cell lung cancer. Mol Biol Rep 39: 3029-3035, 2012.

31. Papas MG, Karatzas PS, Papanikolaou IS, et al: LFA-1 expression in a series of colorectal adenocarcinomas. J Gastrointest Cancer 43: 462-466, 2012.

32. Yamamoto S, Nishimura O, Misaki K, et al: Cthrc1 selectively activates the planar cell polarity pathway of Wnt signaling by stabilizing the Wnt-receptor complex. Dev Cell 15: 23-36, 2008.

33. Veeman MT, Axelrod JD and Moon RT: A second canon. Functions and mechanisms of beta-catenin-independent Wnt signaling. Dev Cell 5: 367-377, 2003.

34. Kikuchi A and Yamamoto H: Tumor formation due to abnormalities in the beta-catenin-independent pathway of Wnt signaling. Cancer Sci 99: 202-208, 2008.

35. Park EH, Kim S, Jo JY, et al: Collagen triple helix repeat containing-1 promotes pancreatic cancer progression by regulating migration and adhesion of tumor cells. Carcinogenesis 34: 694-702, 2013.

36. Chen YL, Wang TH, Hsu HC, Yuan RH and Jeng YM: Overexpression of CTHRC1 in hepatocellular carcinoma promotes tumor invasion and predicts poor prognosis. PLoS One 8: e70324, 2013. 